\title{
Unpolarised TMD PDFs and FFs and the role of transverse momentum dependence in azimuthal spin asymmetries
}

\author{
M. Anselmino ${ }^{a, b}$, M. Boglione ${ }^{a, b}$, U. D’Alesio ${ }^{c, d}$, F. Murgia ${ }^{* d}$ and A. Prokudin ${ }^{e, f}$ \\ ${ }^{a}$ Dipartimento di Fisica, Università di Torino, Via P. Giuria 1, I-10125, Torino, Italy \\ ${ }^{b}$ INFN, Sezione di Torino, Via P. Giuria 1, I-10125, Torino, Italy \\ ${ }^{c}$ Dipartimento di Fisica, Università di Cagliari, Cittadella Universitaria, I-09042 Monserrato \\ (CA), Italy \\ ${ }^{d}$ INFN, Sezione di Cagliari, Cittadella Universitaria, I-09042 Monserrato (CA), Italy \\ e Science Division, Penn State University Berks, Reading, Pennsilvania 19210 USA \\ ${ }^{f}$ Theory Center, Jefferson Lab, 12000 Jefferson Avenue, Newport News, Virginia 23606, Usa \\ Email: mauro.anselminode.infn.it elena.boglionedto.infn.it, \\ umberto.dalesiodca.infn.it francesco.murgiadca.infn.it, \\ prokudindjlab.org
}

In the TMD approach, the average transverse momentum of the unpolarised TMD PDFs and FFs is crucial not only to reproduce unpolarised cross sections and hadron multiplicities, but also for the understanding of azimuthal and spin asymmetries. Information on these transverse momenta is nowadays obtained mainly by fitting multiplicities data for SIDIS, where the intrinsic motion in the initial parton distributions and in the hadronisation process are strongly correlated and difficult to estimate separately without ambiguities. In this contribution we discuss the consequences of this correlation effects on the predictions for the Sivers and Collins asymmetries measured in SIDIS and $e^{+} e^{-}$annihilations, and under active investigation for Drell-Yan processes at RHIC and at CERN by the COMPASS experiment. We show that these effects may be relevant and can sensibly modify the size of the predicted asymmetries. Therefore, they must be taken into careful account when investigating other aspects of TMDs, like the evolution properties of the Sivers and Collins functions and the expected process dependence of the Sivers function.

23rd International Spin Physics Symposium - SPIN2018 -

10-14 September, 2018

Ferrara, Italy

\footnotetext{
* Speaker.
} 


\section{Introduction}

The transverse momentum dependent (TMD) formalism is nowadays the most accredited theoretical approach aiming at explaining a wealth of interesting and puzzling experimental results, collected over the last years, on single spin and azimuthal asymmetries in semi-inclusive deep inelastic scattering (SIDIS), Drell-Yan, and $e^{+} e^{-} \rightarrow h_{1} h_{2}+X$ processes (see e.g. Ref. [四] for an introduction to the subject). In this approach, a new class of transverse momentum dependent parton distributions (TMD PDFs) and fragmentation functions (TMD FFs), also known collectively as TMDs for short, are ultimately responsible for the azimuthal asymmetries measured at the hadronic level. At leading twist, for a spin 1/2 hadron like the proton, there are 8 independent TMD PDFs. Three of them survive in the collinear configuration and correspond to the well-known unpolarised, longitudinally polarised, and transversely polarised quark parton distribution functions. In the fragmentation sector, for (pseudo)scalar particles, like pions and kaons, there are at leading twist only 2 independent TMDs, the unpolarised fragmentation function (surviving in the collinear configuration) and the Collins FF.

Among the TMDs, besides the unpolarised functions, the Sivers distribution [2] and the Collins FF [3] are of special relevance. In fact, they can be responsible, alone or in combination, for many of the most interesting spin and azimuthal asymmetries observed. We will limit our considerations to these two TMDs in the sequel.

A crucial point in the TMD approach is the phenomenological parametrisation of the explicit transverse momentum dependence of TMD PDFs and FFs. To this end, a simple Gaussian shape, or combinations of Gaussian shapes multiplied by appropriate powers of the transverse momentum, are commonly adopted. For unpolarised TMDs, the most relevant parameter is the average square transverse momentum, $\left\langle k_{\perp}^{2}\right\rangle$ and $\left\langle p_{\perp}^{2}\right\rangle$, respectively for PDFs and FFs. The choice of these parameters affects subsequent predictions for the spin and azimuthal asymmetries.

At present most of the information on the unpolarised TMDs, and on the Sivers and Collins functions and the related spin and azimuthal asymmetries, comes from (un)polarised data for SIDIS processes, $\ell p \rightarrow \ell^{\prime} h+X$. However, in this case the transverse momentum dependences of the TMD PDFs and FFs are strongly correlated. In fact, the trasverse momentum of the observed hadron, $\boldsymbol{P}_{T}$, is kinematically related to a combination of $\boldsymbol{k}_{\perp}$ and $\boldsymbol{p}_{\perp}$. In particular, at leading order in a $k_{\perp} / Q$ power expansion (here $k_{\perp}$ is used generically for any intrinsic transverse motion and $Q$ for the large energy scale in the process), $\boldsymbol{P}_{T} \simeq \boldsymbol{p}_{\perp}+z \boldsymbol{k}_{\perp}$, where $z$ is the light-cone momentum fraction in the fragmentation process. As a consequence of this strong correlation, comparably good fits of SIDIS data can be obtained with even very different combinations of the parameters $\left\langle k_{\perp}^{2}\right\rangle$ and $\left\langle p_{\perp}^{2}\right\rangle$. However, these comparable fits to SIDIS data may lead to sensibly different predictions of single spin and azimuthal asymmetries when used in Drell-Yan processes, where only $\boldsymbol{k}_{\perp}$ effects in the initial PDFs are present, or, alternatively, in $e^{+} e^{-} \rightarrow h_{1} h_{2}+X$ processes, where only $\boldsymbol{p}_{\perp}$ effects in the fragmentation sector play a role. In the rest of this contribution we will summarize a detailed analysis aiming at clarifying these aspects of TMD phenomenology.

\section{Theoretical approach}

In order to avoid unessential complications and single out the main qualitative results of inter- 
est here, we work in a simplified scheme. We adopt a TMD factorization approach at leading order and leading twist, considering factorised longitudinal and transverse momentum dependences in all (un)polarised TMDs involved. The longitudinal component is taken proportional to the corresponding unpolarised collinear function times a further $x$ (or $z$ ) dependent term, while for the $k_{\perp}$ (or $p_{\perp}$ ) dependent component we adopt flavour-independent, Gaussian (or Gaussian-like) functional forms, respecting known positivity bounds. Moreover, all (un)polarised cross sections (the numerators and denominators of the spin and azimuthal asymmetries) are integrated over the transverse momentum of the observed hadron(s) (for SIDIS and $e^{+} e^{-}$processes), or of the lepton pair (for Drell-Yan processes) in the range of validity of the TMD approach, $P_{T}, q_{T} \simeq 1-2 \mathrm{GeV} \ll \mathrm{Q}$, where $Q$ refers generically to the large energy scale involved in the process. Notice that from the mathematical point of view, all integrations over $k_{\perp}$ and $p_{\perp}$ are performed in the full range $[0,+\infty)$. Due to the Gaussian shapes adopted, however, the main contribution to the integrals is confined inside the above region of phenomenological interest and validity of the TMD approach.

In this scheme, cross sections and spin/azimuthal asymmetries factorize into a collinear term and a simple, transverse momentum integrated, component. All details and intermediate steps can be found in Ref. [四]. Here we limit ourselves to show the essential ingredients and the final results.

The unpolarised TMD PDFs and FFs are parameterised as follows:

$$
f_{q / p}\left(x, k_{\perp}\right)=f_{q / p}(x) \frac{e^{-k_{\perp}^{2} /\left\langle k_{\perp}^{2}\right\rangle}}{\pi\left\langle k_{\perp}^{2}\right\rangle}, \quad D_{h / q}\left(z, p_{\perp}\right)=D_{h / q}(z) \frac{e^{-p_{\perp}^{2} /\left\langle p_{\perp}^{2}\right\rangle}}{\pi\left\langle p_{\perp}^{2}\right\rangle} .
$$

The quark transversity distribution, the Sivers function and the Collins fragmentation functions are analogously parameterised in the following way:

$$
\begin{gathered}
h_{1}^{q}\left(x, k_{\perp}\right)=h_{1}^{q}(x) \frac{e^{-k_{\perp}^{2} /\left\langle k_{\perp}^{2}\right\rangle_{T}}}{\pi\left\langle k_{\perp}^{2}\right\rangle_{T}}, \\
\Delta^{N} f_{q / p^{\uparrow}}\left(x, k_{\perp}\right)=\Delta^{N} f_{q / p^{\uparrow}}(x) \sqrt{2 e} \frac{k_{\perp}}{M_{S}} e^{-k_{\perp}^{2} / M_{S}^{2}} \frac{e^{-k_{\perp}^{2} /\left\langle k_{\perp}^{2}\right\rangle}}{\pi\left\langle k_{\perp}^{2}\right\rangle} \equiv \Delta^{N} f_{q / p^{\uparrow}}(x) \sqrt{2 e} \frac{k_{\perp}}{M_{S}} \frac{e^{-k_{\perp}^{2} /\left\langle k_{\perp}^{2}\right\rangle_{S}}}{\pi\left\langle k_{\perp}^{2}\right\rangle}, \quad \text { (2.3) } \\
\Delta^{N} D_{h / q^{\uparrow}}\left(z, p_{\perp}\right)=\Delta^{N} D_{h / q^{\uparrow}}(z) \sqrt{2 e} \frac{p_{\perp}}{M_{C}} e^{-p_{\perp}^{2} / M_{C}^{2}} \frac{e^{-p_{\perp}^{2} /\left\langle p_{\perp}^{2}\right\rangle}}{\pi\left\langle p_{\perp}^{2}\right\rangle} \equiv \Delta^{N} D_{h / q^{\uparrow}}(z) \sqrt{2 e} \frac{p_{\perp}}{M_{C}} \frac{e^{-p_{\perp}^{2} /\left\langle p_{\perp}^{2}\right\rangle_{C}}}{\pi\left\langle p_{\perp}^{2}\right\rangle},
\end{gathered}
$$

where

$$
\left\langle k_{\perp}^{2}\right\rangle_{S}=\frac{\left\langle k_{\perp}^{2}\right\rangle M_{S}^{2}}{\left\langle k_{\perp}^{2}\right\rangle+M_{S}^{2}} \quad\left\langle p_{\perp}^{2}\right\rangle_{C}=\frac{\left\langle p_{\perp}^{2}\right\rangle M_{C}^{2}}{\left\langle p_{\perp}^{2}\right\rangle+M_{C}^{2}} .
$$

Of interest here are the transverse momentum integrated expressions of the Sivers asymmetry (for SIDIS and Drell-Yan processes) and of the Collins asymmetry (for SIDIS and $e^{+} e^{-} \rightarrow h_{1} h_{2}+X$ processes). Again, we report below the final results. All details can be found in Ref. [田].

\section{1 $P_{T}$-integrated Sivers asymmetry for the SIDIS process $\ell p^{\uparrow} \rightarrow \ell^{\prime} h+X$}

$$
A_{U T}^{\sin \left(\phi_{h}-\phi_{S}\right)}(x, z)=A_{\mathrm{DIS}}^{S}(x, z) \mathscr{F}_{\mathrm{DIS}}^{S}(z), \quad \text { with } \quad A_{\mathrm{DIS}}^{S}(x, z)=\frac{\sum_{q} e_{q}^{2} \Delta^{N} f_{q / p^{\uparrow}}(x) D_{h / q}(z)}{2 \sum_{q} e_{q}^{2} f_{q / p}(x) D_{h / q}(z)}
$$




$$
\mathscr{F}_{\mathrm{DIS}}^{S}\left(z, \rho_{S}, \xi_{1}\right)=\sqrt{\frac{e \pi}{2}}\left[\frac{\rho_{S}^{3}\left(1-\rho_{S}\right)}{\rho_{S}+\xi_{1} / z^{2}}\right]^{1 / 2}, \quad \xi_{1}=\frac{\left\langle p_{\perp}^{2}\right\rangle}{\left\langle k_{\perp}^{2}\right\rangle}, \quad \rho_{S}=\frac{\left\langle k_{\perp}^{2}\right\rangle_{S}}{\left\langle k_{\perp}^{2}\right\rangle}=\frac{1}{1+\frac{\left\langle k_{\perp}^{2}\right\rangle}{M_{S}^{2}}}
$$

2.2 $P_{T}$-integrated Collins asymmetry for the SIDIS process $\ell p^{\uparrow} \rightarrow \ell^{\prime} h+X$

$$
\begin{aligned}
& A_{U T}^{\sin \left(\phi_{h}+\phi_{S}\right)}(x, y, z)=A_{\mathrm{DIS}}^{C}(x, y, z) \mathscr{F}_{\mathrm{DIS}}^{C}(z), \quad A_{\mathrm{DIS}}^{C}(x, y, z)=\frac{1-y}{1+(1-y)^{2}} \frac{\sum_{q} e_{q}^{2} h_{1}^{q}(x) \Delta^{N} D_{h / q^{\uparrow}}(z)}{\sum_{q} e_{q}^{2} f_{q / p}(x) D_{h / q}(z)} \\
& \mathscr{F}_{\mathrm{DIS}}^{C}\left(z, \rho_{C}, \xi_{1} / \xi_{T}\right)=\sqrt{\frac{e \pi}{2}}\left[\frac{\rho_{C}^{3}\left(1-\rho_{C}\right)}{\rho_{C}+z^{2}\left(\xi_{T} / \xi_{1}\right)}\right]^{1 / 2}, \quad \xi_{T}=\frac{\left\langle k_{\perp}^{2}\right\rangle_{T}}{\left\langle k_{\perp}^{2}\right\rangle}, \quad \rho_{C}=\frac{\left\langle p_{\perp}^{2}\right\rangle_{C}}{\left\langle p_{\perp}^{2}\right\rangle}=\frac{1}{1+\frac{\left\langle p_{\perp}^{2}\right\rangle}{M_{C}^{2}}} .
\end{aligned}
$$

2.3 $P_{T}$-integrated Sivers asymmetry for the Drell-Yan process $h_{1}^{\uparrow} h_{2} \rightarrow \ell^{-} \ell^{+}+X$

$$
\begin{aligned}
& A_{N}^{\mathrm{DY}}(y, M)=A_{\mathrm{DY}}^{S}\left(x_{1}, x_{2}\right) \mathscr{F}_{\mathrm{DY}}^{S}, \quad A_{\mathrm{DY}}^{S}\left(x_{1}, x_{2}\right) \equiv A_{\mathrm{DY}}^{S}(y, M)=\frac{\sum_{q} e_{q}^{2} \Delta^{N} f_{q / h_{1}^{\uparrow}}\left(x_{1}\right) f_{\bar{q} / h_{2}}\left(x_{2}\right)}{2 \sum_{q} e_{q}^{2} f_{q / h_{1}}\left(x_{1}\right) f_{\bar{q} / h_{2}}\left(x_{2}\right)}, \\
& \mathscr{F}_{\mathrm{DY}}^{S}\left(\rho_{S}, \xi_{21}\right)=\sqrt{\frac{e \pi}{2}}\left[\frac{\rho_{S}^{3}\left(1-\rho_{S}\right)}{\rho_{S}+\xi_{21}}\right]^{1 / 2}, \quad \xi_{21}=\frac{\left\langle k_{\perp 2}^{2}\right\rangle}{\left\langle k_{\perp 1}^{2}\right\rangle}, \quad \rho_{S}=\frac{\left\langle k_{\perp}^{2}\right\rangle_{S}}{\left\langle k_{\perp 1}^{2}\right\rangle}=\frac{1}{1+\frac{\left\langle k_{11}^{2}\right\rangle}{M_{S}^{2}}} .
\end{aligned}
$$

2.4 $P_{T}$-integrated Collins asymmetry for $e^{+} e^{-} \rightarrow h_{1} h_{2}+X$ (hadronic-plane frame)

$$
\begin{gathered}
P_{0}^{h_{1} h_{2}}\left(z_{1}, z_{2} ; \theta\right)=A_{\mathrm{ee}}^{h_{1} h_{2}}\left(z_{1}, z_{2} ; \theta\right) \mathscr{F}_{\mathrm{ee}}^{C}, \quad \mathscr{F}_{\mathrm{ee}}^{C}\left(\rho_{C}\right)=2 e \rho_{C}^{2}\left(1-\rho_{C}\right), \\
A_{\mathrm{ee}}^{h_{1} h_{2}}\left(z_{1}, z_{2} ; \theta\right)=\frac{1}{4} \frac{\sin ^{2} \theta}{1+\cos ^{2} \theta} \frac{z_{1} z_{2}}{z_{1}^{2}+z_{2}^{2}} \frac{\sum_{q} e_{q}^{2} \Delta^{N} D_{h_{1} / q^{\uparrow}}\left(z_{1}\right) \Delta^{N} D_{h_{2} / \bar{q}^{\uparrow}}\left(z_{2}\right)}{\sum_{q} e_{q}^{2} D_{h_{1} / q}\left(z_{1}\right) D_{h_{2} / \bar{q}}\left(z_{2}\right)} .
\end{gathered}
$$

Notice that for simplicity we are assuming here that the hadrons $h_{1,2}$ are both pions or kaons. Cases like $\pi K$ pairs would in general require two different values of $\left\langle p_{\perp}^{2}\right\rangle$. Similar results can be obtained adopting the thrust-axis frame.

\section{Phenomenological results}

In order to clarify our discussion and reduce the number of free parameters, we will perform some additional simplifying assumptions: a) Concerning the transversity distribution, we will assume that $\left\langle k_{\perp}^{2}\right\rangle_{T}=\left\langle k_{\perp}^{2}\right\rangle$, that is $\left.\xi_{T} \equiv 1 ; \mathrm{b}\right)$ We only consider Drell-Yan processes in proton-proton collisions; this amounts to take $\left\langle k_{\perp 1}^{2}\right\rangle=\left\langle k_{\perp 2}^{2}\right\rangle \equiv\left\langle k_{\perp}^{2}\right\rangle$, and $\xi_{21} \equiv 1$ in the sequel; The inclusion of the COMPASS case, for DY in $\pi p$ collisions, would require two independent values for the average square transverse momentum in the initial pion and proton beams.

In this simplified but realistic scheme, the transverse momentum integrated components of the asymmetries (the functions $\mathscr{F}^{S, C}$ in Eqs. ([2.6) $($ (2.13])) depend only on three parameters: 1) 
$\xi_{1}=\left\langle p_{\perp}^{2}\right\rangle /\left\langle k_{\perp}^{2}\right\rangle$, estimated by fitting unpolarised observables (multiplicities, the Cahn effect); 2) $\rho_{S, C}$, that are fixed, using $\xi_{1}$, by fitting available data on spin/azimuthal asymmetries. Notice that in the SIDIS case there is a residual dependence on $z$, the light-cone momentum fraction in the fragmentation process.

A few comments on these crucial parameters are in order here. From the mathematical point of view, $0<\xi_{1}<+\infty$, the lower (upper) limit corresponding respectively to a completely collinear configuration in the fragmentation (distribution) sector. Physically, these limiting values are quite extreme and highly unrealistic. A phenomenologically plausible range of values is $0.15<\xi_{1}<2.2$ (see e.g. Fig. 9 of Ref. [可]). Concerning the parameters $\rho_{S, C}$, they govern, in our scheme, the transverse momentum dependence of the Sivers and Collins functions w.r.t. that of the corresponding unpolarised functions. Mathematically $0<\rho_{S, C}<1$, however also in this case the limiting values are phenomenologically unrealistic.

Now the crucial point of our analysis is the following: How do our predictions on the Sivers and Collins asymmetries in SIDIS, Drell-Yan and $e^{+} e^{-}$annihilation processes depend on the choice of specific values for $\xi_{1}$ and consequently $\rho_{S, C}$ ?

The most general way to answer this question is to study how the transverse components of the asymmetries, the $\mathscr{F}^{S, C}$ functions, change when we move along a generic trajectory in the $\left(\xi_{1}, \rho\right)$ parameter space, starting from some fixed point $\left(\hat{\xi}_{1}, \hat{\rho}\right)$, corresponding to a phenomenological reference fit of unpolarised observables, and subject to possible constraints dictated by the available experimental data on spin/azimuthal asymmetries. In particular, we want to study how these changes reflect on the collinear components of the Sivers and Collins functions and, ultimately, on our predictions for the corresponding asymmetries.

\subsection{The Sivers asymmetry in SIDIS and Drell-Yan processes}

Let us to this end concentrate first on the Sivers case, and consider, to be definite, two different and comparably good parameterisations of the quark Sivers functions delivered by the CagliariTorino group:

1. The fit of Ref. [目], referred as FIT09 in the sequel, for which

$$
\left\langle k_{\perp}^{2}\right\rangle=0.25 \mathrm{GeV}^{2}, \quad\left\langle p_{\perp}^{2}\right\rangle=0.20 \mathrm{GeV}^{2}, \quad M_{S}^{2}=0.34 \mathrm{GeV}^{2},
$$

that implies

$$
\hat{\xi}_{1}^{(09)}=0.80, \quad \hat{\rho}_{S}^{(09)}=0.58 .
$$

2. The fit of Ref. [U], referred as FIT16 in the sequel, for which

$$
\left\langle k_{\perp}^{2}\right\rangle=0.57 \mathrm{GeV}^{2}, \quad\left\langle p_{\perp}^{2}\right\rangle=0.12 \mathrm{GeV}^{2}, \quad M_{S}^{2}=0.80 \mathrm{GeV}^{2},
$$

implying

$$
\hat{\xi}_{1}^{(16)}=0.21, \quad \hat{\rho}_{S}^{(16)}=0.58
$$

All details on the fitting procedures adopted and on the extraction of all parameters (including those for the collinear component of the quark Sivers functions, not mentioned here) can be found in the quoted references. 
Notice that we can always reformulate the general expressions of the functions $\mathscr{F} S, C\left(\xi_{1}, \rho\right)$ in the $\left(\xi_{1}, \rho\right)$ parameter space through a rescaling factor times their value in the fixed starting point, $\hat{\mathscr{F}} S, C\left(\hat{\xi}_{1}, \hat{\rho}\right)$. More in detail, for the asymmetries of interest, we can write:

$$
\begin{gathered}
\mathscr{F}_{\mathrm{DIS}}^{S}\left(z, \xi_{1}, \rho_{S}\right)=R_{\mathrm{DIS}}^{S} \hat{\mathscr{F}}_{\mathrm{DIS}}^{S}\left(z, \hat{\xi}_{1}, \hat{\rho}_{S}\right), \quad R_{\mathrm{DIS}}^{S}=\left[\frac{\rho_{S}^{3}\left(1-\rho_{S}\right)}{\rho_{S}+\xi_{1} / z^{2}} \frac{\hat{\rho}_{S}+\hat{\xi}_{1} / z^{2}}{\hat{\rho}_{S}^{3}\left(1-\hat{\rho}_{S}\right)}\right]^{1 / 2} \\
\mathscr{F}_{\mathrm{DY}}^{S}\left(\xi_{21}=1, \rho_{S}\right)=R_{\mathrm{DY}}^{S} \hat{\mathscr{F}}_{\mathrm{DY}}^{S}\left(\hat{\rho}_{S}\right), \quad R_{\mathrm{DY}}^{S}=\left[\frac{\rho_{S}^{3}\left(1-\rho_{S}\right)}{\rho_{S}+1} \frac{\hat{\rho}_{S}+1}{\hat{\rho}_{S}^{3}\left(1-\hat{\rho}_{S}\right)}\right]^{1 / 2} \\
\mathscr{F}_{\mathrm{DIS}}^{C}\left(z, \xi_{1}, \rho_{C}\right)=R_{\mathrm{DIS}}^{C} \hat{\mathscr{F}}_{\mathrm{DIS}}^{C}\left(z, \hat{\xi}_{1}, \hat{\rho}_{C}\right), \quad R_{\mathrm{DIS}}^{C}=\left[\frac{\rho_{C}^{3}\left(1-\rho_{C}\right)}{\rho_{C}+z^{2} / \xi_{1}} \frac{\hat{\rho}_{C}+z^{2} / \hat{\xi}_{1}}{\hat{\rho}_{C}^{3}\left(1-\hat{\rho}_{C}\right)}\right]^{1 / 2} \\
\mathscr{F}_{\mathrm{ee}}^{C}\left(\rho_{C}\right)=R_{\mathrm{ee}}^{C} \hat{\mathscr{F}}_{\mathrm{ee}}^{C}\left(\hat{\rho}_{C}\right), \quad R_{\mathrm{ee}}^{C}=\frac{\rho_{C}^{2}\left(1-\rho_{C}\right)}{\hat{\rho}_{C}^{2}\left(1-\hat{\rho}_{C}\right)} .
\end{gathered}
$$

Let us now consider in more detail the Sivers case. Almost all data available come from SIDIS, mainly from the HERMES and COMPASS experiments. Therefore, we will assume that all acceptable trajectories in the $\left(\xi_{1}, \rho_{S}\right)$ parameter space are bound to preserve the value of the total Sivers asymmetry:

$$
A_{\mathrm{DIS}}^{S}(x, z) \mathscr{F}_{\mathrm{DIS}}^{S}\left(z, \xi_{1}, \rho_{S}\right) \simeq \hat{A}_{\mathrm{DIS}}^{S}(x, z) \hat{\mathscr{F}}_{\mathrm{DIS}}^{S}\left(z, \hat{\xi}_{1}, \hat{\rho}_{S}\right)
$$

or, equivalently,

$$
\mathscr{F}_{\mathrm{DIS}}^{S}=R_{\mathrm{DIS}}^{S} \hat{\mathscr{F}}_{\mathrm{DIS}}^{S}, \quad A_{\mathrm{DIS}}^{S} \simeq \frac{1}{R_{\mathrm{DIS}}^{S}} \hat{A}_{\mathrm{DIS}}^{S} \quad \text { with } \quad R_{\mathrm{DIS}}^{S}=\left[\frac{\rho_{S}^{3}\left(1-\rho_{S}\right)}{\rho_{S}+\xi_{1} / z^{2}} \frac{\hat{\rho}_{S}+\hat{\xi}_{1} / z^{2}}{\hat{\rho}_{S}^{3}\left(1-\hat{\rho}_{S}\right)}\right]^{1 / 2},
$$

On the contrary, since only very few, low-statistic data are presently available for the Sivers asymmetry in Drell-Yan processes, they are not used in the fits of the Sivers function. As a result, the full DY Sivers asymmetry is not constrained like the SIDIS one. However, given that both the SIDIS and DY asymmetries depend linearly on the Sivers function, we may reasonably assume that

$$
\frac{A_{\mathrm{DY}}^{S}}{\hat{A}_{\mathrm{DY}}^{S}} \simeq \frac{A_{\mathrm{DIS}}^{S}}{\hat{A}_{\mathrm{DIS}}^{S}} \quad \Rightarrow A_{\mathrm{DY}}^{S} \simeq\left(\frac{A_{\mathrm{DIS}}^{S}}{\hat{A}_{\mathrm{DIS}}^{S}}\right) \hat{A}_{\mathrm{DY}}^{S} \equiv \frac{1}{R_{\mathrm{DIS}}^{S}} \hat{A}_{\mathrm{DY}}^{S} .
$$

Bearing in mind Eq. (3.6), we can then write the full DY asymmetry as

$$
A_{N}^{\mathrm{DY}}=A_{\mathrm{DY}}^{S} \mathscr{F}_{\mathrm{DY}}^{S} \simeq\left(\frac{R_{\mathrm{DY}}^{S}}{R_{\mathrm{DIS}}^{S}}\right) \hat{A}_{\mathrm{DY}}^{S} \hat{\mathscr{F}}_{\mathrm{DY}}^{S}=R_{\mathrm{DY}}^{N} \hat{A}_{N}^{\mathrm{DY}}, \quad \text { with } \quad R_{\mathrm{DY}}^{N}=\left[\frac{\rho_{S}+\xi_{1} / z^{2}}{\hat{\rho}_{S}+\hat{\xi}_{1} / z^{2}} \frac{\hat{\rho}_{S}+1}{\rho_{S}+1}\right]^{1 / 2} .
$$

Now, let us come back to the two reference fits for the quark Sivers functions, FIT09 and FIT16, see respectively Eqs. (B.2) and (B.4). As we said, both sets reproduce comparably well the SIDIS Sivers asymmetry data. Notice however that, while $\hat{\rho}_{S}^{(09)} \simeq \hat{\rho}_{S}^{(16)} \simeq \hat{\rho}_{S} \simeq 0.58$, due to the 
strong correlation between $\left\langle k_{\perp}^{2}\right\rangle$ and $\left\langle p_{\perp}^{2}\right\rangle$ and the corresponding uncertainties, the values of $\hat{\xi}_{1}^{(09)}$ and $\hat{\xi}_{1}^{(16)}$ are sizably different. From Eq. (B.12), we therefore see that the predictions of the two reference sets for the Sivers asymmetry in Drell-Yan processes are related as follows:

$$
A_{N}^{\mathrm{DY}}\left(\hat{\rho}_{S}, \hat{\xi}_{1}^{(16)}\right) \simeq\left[\frac{\hat{\rho}_{S}+\hat{\xi}_{1}^{(16)} / z^{2}}{\hat{\rho}_{S}+\hat{\xi}_{1}^{(09)} / z^{2}}\right]^{1 / 2} \hat{A}_{N}^{\mathrm{DY}}\left(\hat{\rho}_{S}, \hat{\xi}_{1}^{(09)}\right)
$$

Using the values for the $\hat{\xi}_{1}, \hat{\rho}_{S}$ parameters given above for the two sets, one finds that the rescaling factor in Eq. (B.13) varies in the range $[0.52,0.68]$ for $0.1<z<0.7$. Since the SIDIS data utilized in the fits are dominated by the small- $z$ region, we find that

$$
A_{N}^{\mathrm{DY}}\left(\hat{\rho}_{S}, \hat{\xi}_{1}^{(16)}\right) \simeq \frac{1}{2} \hat{A}_{N}^{\mathrm{DY}}\left(\hat{\rho}_{S}, \hat{\xi}_{1}^{(09)}\right) .
$$

The main outcome of this study is therefore that, because of the unavoidable strong correlations between the parameters $\left\langle k_{\perp}^{2}\right\rangle$ and $\left\langle p_{\perp}^{2}\right\rangle$, comparably good fits to the Sivers asymmetry in SIDIS may lead to very different estimates for the same asymmetries in Drell-Yan processes. Since the Drell-Yan process is crucial in order to study the process dependence and the evolution properties of the Sivers function, this effect should be taken into careful account, before drawing any conclusion concerning these aspects.

The formalism presented in the previous pages can be generalised and applied to different scenarios for the Sivers effect and to the Collins asymmetry in SIDIS and $e^{+} e^{-}$annihilation processes.

As an example, instead of fixing the full SIDIS Sivers asymmetry as a whole, when enough data on the transverse momentum dependence of the asymmetry will be available, one could think of fixing separately both the collinear $\left(A_{\text {DIS }}^{S}\right.$ ) and the $P_{T}$-integrated ( $\mathscr{F}_{\text {DIS }}^{S}$ ) components of the asymmetry. From Eq. (B.TO), we see that this corresponds to require $R_{\mathrm{DIS}}^{S}=1$ (the Sivers scenario 2 of Ref. [田]). Under this constraint, the allowed values of $\rho_{S}$ as a function of $\xi_{1}$ for $z=0.2$, and the resulting rescaling factor for the Drell-Yan Sivers asymmetry, are shown respectively in Figs. ए and ๒].
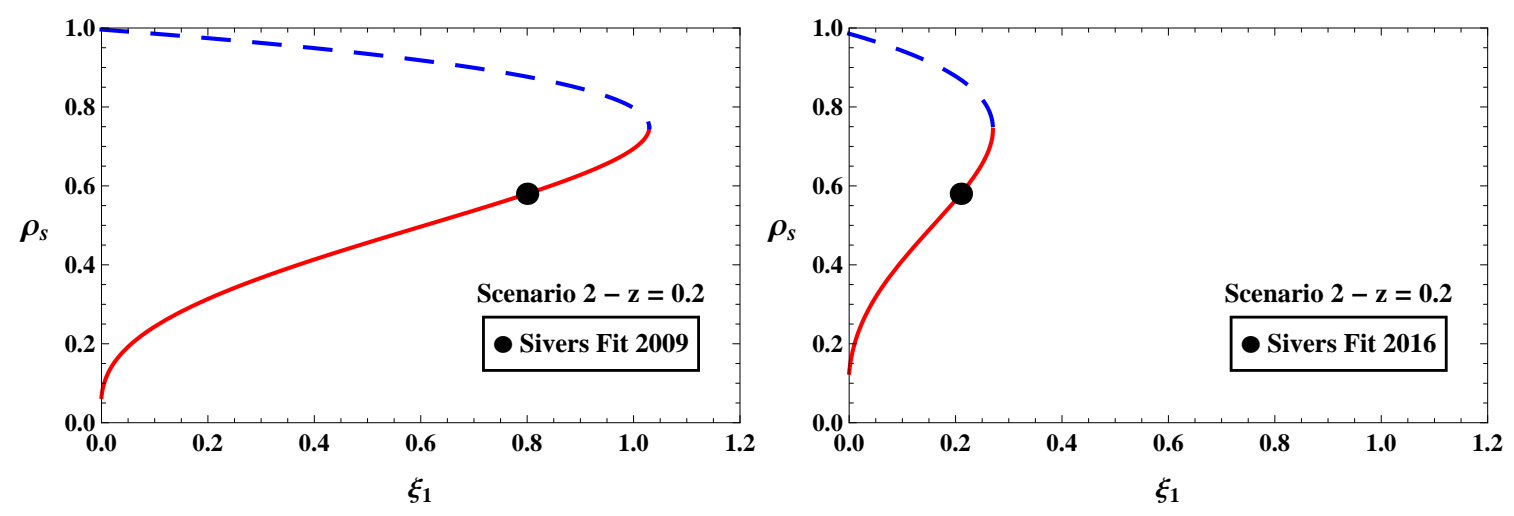

Figure 1: Sets of values of $\rho_{S}$ and $\xi_{1}$ which leave unchanged $\mathscr{F}_{\text {DIS }}^{S}(z=0.2)$, see Eq. (‥ $)$. The black dots correspond to the fits of Ref. [四] (left plot, FIT09) and of Ref. [四] (right plot, FIT16). Notice that for each value of $\xi_{1}$ one finds two possible values of $\rho_{S}$. Similar results are found for $z=0.4$ or 0.6. 

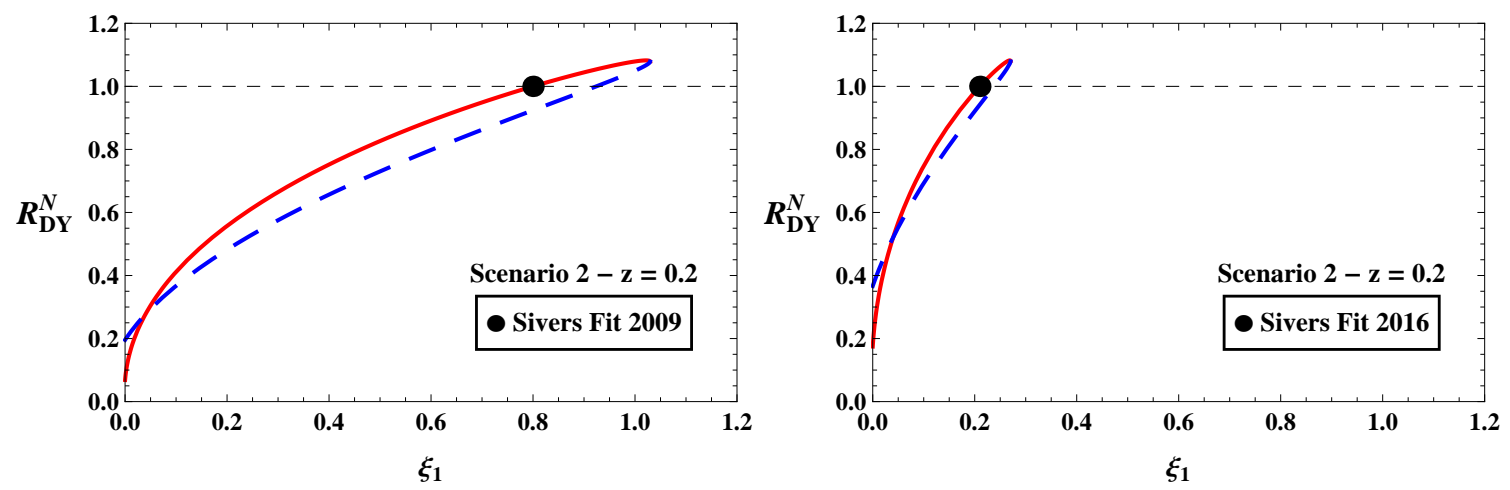

Figure 2: Change in the Drell-Yan $q_{T}$-integrated Sivers asymmetry $A_{N}^{\mathrm{DY}}$, Eqs. (미) and (․ㅣ), as functions of $\xi_{1}$, in correspondence of the $\rho_{S}$ values shown in Fig. . The rescaling factor $R_{\mathrm{DY}}^{N}$ is defined in Eq. (B.T2). In this scenario $A_{U T}^{\sin \left(\phi_{h}-\phi_{S}\right)}$, Eq. (2.6), does not change, together with its components $A_{\text {DIS }}^{S}$ and $\mathscr{F}$ DIS.

Hopefully, in the near future, when more data on the DY Sivers asymmetry will be available from COMPASS and RHIC, one could try to constrain the allowed values of the $\xi_{1}$ and $\rho_{S}$ parameters by requiring to reproduce both the SIDIS and Drell-Yan Sivers asymmetries at the same time. These more general scenarios are discussed in depth in Ref. [四], to which we refer the reader for further details.

\subsection{The Collins asymmetry in SIDIS and $e^{+} e^{-}$annihilation processes}

The treatment of the Collins asymmetry is more complicated than the Sivers case. First of all, the Collins fragmentation function enters linearly in the SIDIS Collins asymmetry, convoluted with the transversity distribution, while it appears "quadractically" (as a convolution of two Collins FFs) in the $e^{+} e^{-}$case. Therefore, changes in the value of $\xi_{1}$ can affect only the transversity distribution, or only the Collins function or, more probably, both of them simultaneously, leaving room to more different possible scenarios. Moreover, in contrast to the Sivers case, detailed experimental information is available both for SIDIS and $e^{+} e^{-}$processes.

Also in this case, we have considered two comparable reference fits for the transversity distribution and the Collins FF with similar values of $\rho_{C}$ but very different values of $\xi_{1}$, see Ref. [田]. The detailed analysis of several possible scenarios, covering all possibilities mentioned above, shows that also in the Collins case the estimates of the collinear components of the transversity distribution and the Collins function may vary as a function of $\xi_{1}$. However, these changes seem to be milder than in the Sivers case, apart from some marginal configurations (see Ref. [四] for more details).

\section{Concluding remarks}

All present parameterisations of the most interesting and most studied TMDs, the quark transversity and Sivers distributions and the Collins fragmentation function, originate mainly from SIDIS data, to some extent from $e^{+} e^{-}$data and only marginally from Drell-Yan results.

In this contribution we have investigated, in a simple but general TMD approach, to what extent the unavoidable strong correlations between the average transverse momenta for the TMD 
PDFs and FFs, as estimated from unpolarized SIDIS data, may affect the extraction of the collinear component of the polarized TMDs and, ultimately, the predictions for spin/azimuthal asymmetries in Drell-Yan and $e^{+} e^{-} \rightarrow h_{1} h_{2}+X$ processes.

We have shown that comparably good fits of the SIDIS Sivers and Collins azimuthal asymmetries can be obtained with (even very) different values of the ratio $\xi_{1}=\left\langle p_{\perp}^{2}\right\rangle /\left\langle k_{\perp}^{2}\right\rangle$. As a consequence, the corresponding estimates for the Sivers asymmetry in Drell-Yan processes can differ by a factor of up to 2 .

Concerning the extraction of the Collins fragmentation function and of the transversity distribution from SIDIS and $e^{+} e^{-}$annihilation data, the uncertainty on $\xi_{1}$ seems to have milder (but still not negligible) effects, except for some marginal cases.

This analysis shows that a more precise knowledge of $\left\langle k_{\perp}^{2}\right\rangle,\left\langle p_{\perp}^{2}\right\rangle$ and $\xi_{1}$ is crucial. This is particularly true since we are entering a new stage in the exploration of the $3 \mathrm{D}$ structure of hadrons, aiming at a more precise determination of the functional shapes of the TMD PDFs and FFs, the understanding of their process dependence and a full implementation of TMD evolution. For this, new experimental results from RHIC, Jlab, COMPASS and the future planned Electron Ion Collider will be crucial.

\section{References}

[1] U. D'Alesio and F. Murgia, Azimuthal and Single Spin Asymmetries in Hard Scattering Processes, Prog. Part. Nucl. Phys. 61 (2008) 394 [0712. 4328].

[2] D. W. Sivers, Single spin production asymmetries from the hard scattering of point - like constituents, Phys. Rev. D41 (1990) 83.

[3] J. C. Collins, Fragmentation of transversely polarized quarks probed in transverse momentum distributions, Nucl. Phys. B396 (1993) 161.

[4] M. Anselmino, M. Boglione, U. D'Alesio, F. Murgia and A. Prokudin, Role of transverse momentum dependence of unpolarized parton distribution and fragmentation functions in the analysis of azimuthal spin asymmetries, Phys. Rev. D98 (2018) 094023 [11809.09500].

[5] A. Bacchetta, F. Delcarro, C. Pisano, M. Radici and A. Signori, Extraction of partonic transverse momentum distributions from semi-inclusive deep-inelastic scattering, Drell-Yan and Z-boson production, [HEP 06 (2017) 081] [प103.1015]].

[6] M. Anselmino, M. Boglione, U. D’Alesio, A. Kotzinian, S. Melis, F. Murgia et al., Sivers Effect for Pion and Kaon Production in Semi-Inclusive Deep Inelastic Scattering, Eur. Phys. J. A39 (2009) 89 [0805.2677].

[7] M. Anselmino, M. Boglione, U. D'Alesio, F. Murgia and A. Prokudin, Study of the sign change of the Sivers function from STAR Collaboration W/Z production data, [HEP 04 (2017) 046 [15612.06413]. 